\title{
Effects of pleasant visual stimulation on attention, working memory, and anxiety in college students
}

\author{
Débora Gago and Rosa Maria Martins de Almeida \\ Universidade Federal do Rio Grande do Sul, Porto Alegre, RS, Brazil
}

\begin{abstract}
Different emotional reactions can be induced by the presentation of visual stimuli with affective content. Emotional stimuli are processed and linked with cognitive functions, such as attention, memory, and anxiety, and have implications in the mental health field. Previous studies have reported that positive and negative emotions tend to change cognitive processes in individuals, ultimately resulting in better and worse performance, respectively. Many studies have emphasized the crucial role of affect in directing attention to relevant stimuli, enhancing learning and memory, facilitating decision making, selecting goals, and conflict resolution. The aim of the present study was to investigate the influence of pleasant visual stimuli on memory, focused attention, and anxiety and further understand the effects of emotional induction. The study investigated the effects of presenting a pleasant visual stimulus in a 1.5 min video to a sample of 145 college students on focused attention, working memory (Personnel Selection Testing, memory subtest), and anxiety (State-Trait Anxiety Inventory). Nonsignificant differences were observed in focused attention, working memory, and anxiety state. Statistically significant differences were found in trait anxiety and the comparison between men and women with regard to memory and anxiety. The positive stimulus was not sufficient to alter cognition or emotion in our research participants. Emotion was found to not be the only factor that influences memory, and other factors appear to be important, such as prior knowledge and cognitive, social, and physiological factors, including personal history, the environment, and culture. Keywords: stimulus, emotion, cognition, neuropsychology.
\end{abstract}

Received 18 May 2013; received in revised form 01 September 2013; accepted 02 September 2013. Available online 23 December 2013.

\section{Introduction}

Emotion is the main element involved in the positive and negative reinforcement of behavioral choices. It plays a crucial role in the adoption of strategies that result in a higher probability of wellbeing for individuals and their associates (Moll, Souza, Miranda, Bramati, Veras, \& Magalhães, 2001). Emotion can be defined as specific, consistent collections of cognitive and physiological responses triggered by brain systems that prepare the body for social action and interaction (Damásio, 2000; Lang, 1995; Lazarus, 1993). Emotional experience can be formalized by three components: valence (positive and negative), awareness, and motor activation

Débora Gago and Rosa Maria Martins de Almeida, Instituto de Psicologia, Universidade Federal do Rio Grande do Sul, Porto Alegre, Brazil. Maria Martins de Almeida, Instituto de Psicologia do Desenvolvimento e da Personalidade da Universidade Federal do Rio Grande do Sul (UFRGS), Porto Alegre, RS, Brazil. Laboratório de Psicologia Experimental, Neurociências e Comportamento (LPNeC). Pesquisadora de Produtividade do CNPq 1D. Correspondence regarding this article should be directed to: Rosa Maria Martins de Almeida, Rua Ramiro Barcelos n. 2600, Porto Alegre, CEP: 90035-003, Brazil. E-mail: rosa_almeida@yahoo.com or rosa.almeida@ufrgs.br (approach and avoidance). Emotional valence can also be addressed as two motivational systems: one related to negative (aversive) emotions and another related to positive (approach) emotions. In the aversion system, an aversive stimulus generates negative affect, thus facilitating the behavior of retraction. Fear and disgust are examples of stimuli related to this system; they are associated with an increased distance between the individual and the source that generates the stimulus. The approach system, in contrast, is related to positive emotional experiences. In this case, the stimulus induces approach behavior (Heilman, 1997).

Emotional aspects are important factors in memory because they may affect recall and recognition processes based on encoding processes. Emotional processes at the neurobiological level are mediated by the amygdala. When activated, it mediates anatomical connections with the cortex that can facilitate the processing of any stimuli that are presented. Additionally, anatomical connections between the amygdala and hippocampus can directly influence semantic memory. Thus, greater activation in the amygdala at the time of learning is associated with a greater intensity of the stored memory of the facts that have emotional content. Furthermore, evidence indicates that the congruence of emotional aspects at the time of encoding and subsequent memory recall increases the ability to recall facts (Pergher, Grassi-Oliveira, Ávila, \& Stein, 2005). 
Stimuli with emotional content are best recovered in tests of recall and recognition than stimuli without emotional content. The same is true for attention (Buchanan, Denburg, Tranel, \& Adolphs, 2001; LaBar, \& Phelps, 1998; Ochsner, 2000; Kensinger, \& Corkin, 2003). Emotion is not the only factor that influences memory. Other key factors play important roles, such as prior knowledge and the development of cognitive processes, and anatomical, physiological, and social factors, such as personal history, the environment, and the culture in which the individual has grown up and lived. Emotion, in turn, does not affect only memory. When faced with an intense emotional state, not only is memory affected. The entire cognitive system is impacted, with repercussions at the levels of perception, attention, memory, reasoning, language, and decision-making (Christianson, 1992; Schacter, Kagan, \& Leichtman, 1995).

In a laboratory setting, inducing intense negative experiences is easier, faster, and more uniform than inducing positive experiences or negative emotional experiences of accidents and injuries or positive emotional experiences with anecdotes, descriptions, or erotic comedy movies. The classification of negative experiences also has greater uniformity than the classification of positive experiences (Pinto, 1998).

An important focus of studies on emotional modulation is the analysis of individual variability in the processing of emotional stimuli (Kosslyn et al., 2002). Affective bias is an important feature of emotion because the same event can stimulate different emotional reactivity patterns in different individuals (Davidson, Jackson, \& Kalin 2000; Davidson, 2003).

Studies have linked memory with dopamine and provided evidence that the release of this neurotransmitter increases when positive emotions are induced, with a direct impact on the formation of memories in the hippocampus (Shohamy, \& Adcock, 2010). However, some authors have argued that positive mood can undermine people's performance on tests of executive function (Oaksford, Morris, Grainger, \& Williams, 1996). This was verified by Oaksford et al. (1996), who analyzed the performance of people in the Tower of London task, which is described as a classic instrument for measuring executive function. Phillips, Bull, Adams, and Fraser (2002) investigated the effects of positive mood on the Stroop test and a fluency test, which are often used to assess executive function. The results of this experiment showed that positive mood increased performance with regard to creativity in the fluency test, however, there is some evidence that it may impair executive functioning. These authors claimed that the effect of inducing positive mode on a test of executive function can ultimately vary according to which test is administered (i.e., depending on whether the test is motivating).

Some authors have argued that emotional stimuli have priority in the processing of attention and memory because of the effect of excitation caused by the stimulus (Murphy, Hill, Ramponi, Calder, \& Barnard, 2010). These authors highlighted the fact that many experiments have found that people pay more attention to stimuli with emotional content than neutral stimuli. This occurs because of the adaptive significance of these stimuli. Notably, excitation can be caused by a stimulus, which can influence attention, regardless of whether the stimulus has positive or negative valence. A positive or negative image can cause the same kind of excitement compared with neutral images, thus producing the same level of attention. Furthermore, Dreisbach and Goschke (2004) argued that positive emotion reduces perseverance when an individual needs to divert attention from one stimulus to another. However, they also emphasized that positive emotion can increase distraction.

With regard to memory, emotional aspects are important factors because they may affect recall and recognition processes based on encoding processes. Such emotional processes at the neurobiological level are mediated by the amygdala. When activated, the amygdala mediates anatomical connections with the cortex, which may facilitate the processing of any stimuli that are presented. Furthermore, anatomical connections between the amygdala and hippocampus can directly influence semantic memory. Thus, when the amygdala is more active at the time of learning, the intensity of this stored memory is greater for events that have emotional content. Furthermore, evidence indicates that emotional congruence when encoding and subsequently retrieving memory increases the ability to recall facts (Pergher et al., 2006).

With regard to anxiety, some authors have argue that it impairs the ability to think and concentrate, suggesting that the interaction between emotion and cognition may elucidate the debilitating nature of pathological anxiety (Vytal, Cornwell, Arkin, \& Grillon, 2012). Although anxiety is adaptive, it can also be debilitating by interfering with our daily life and goaldirected behaviors. Some of the prominent cognitive problems associated with anxiety are mediated by mechanisms of impaired attention. Anxious people complain of being easily distracted and have difficulty concentrating. Population-based studies have reported impairments in executive function and episodic memory across various anxiety disorders (Airaksinen, Larsson, \& Forsell, 2005).

Considering the current evidence of the relationship between cognition and the processing of emotional stimuli, the present study investigated the influence of pleasant visual stimuli on memory, focused attention, and anxiety to contribute to our understanding of the effects of pleasant visual stimuli on emotional induction.

\section{Methods}

This research was conducted using a quantitative, cross-sectional design because of the analytical and data collection procedures.

\section{Participants}

The present study included a total of 145 participants of both sexes, aged 18 to 69 years (mean, 21.8 years; 
standard deviation $[S D], 6.31$ years). The participants were divided into three groups. The first group received no visual stimulus $(n=49)$. The second group received a neutral visual stimulus $(n=47)$. The third group received a pleasant visual stimulus $(n=49)$.

In this study, $35.9 \%$ of the participants were male $(n=52)$, and $64.1 \%$ were female $(n=93)$. The majority of the participants were socioeconomically classified as class $\mathrm{C}$ (income between $\mathrm{R} \$ 2,488$ and $\mathrm{R} \$ 6,220$; Standard Deviation $=1 ; 26)$.

In group 1 (no visual stimulus), $63 \%$ of the 49 participants were female $(n=31)$, and $37 \%$ were male $(n=18)$. In group 2 (neutral stimulus), 64\% of the 47 participants were female $(n=30)$, and $36 \%$ were male $(n=17)$. In group 3 (pleasant visual stimulus), $65 \%$ of the 49 participants were female $(n=32)$, and $37 \%$ were male $(n=17$; Table 1$)$. We attempted to pair the number of men and women but could not because more women participated than men.

Table 1. Description of groups, percentages of men and women in each group, and total number $(n)$ of participants.

\begin{tabular}{lcccccc}
\hline \multirow{2}{*}{ Group } & \multicolumn{5}{c}{ Sex } & Total \\
\cline { 2 - 6 } & \multicolumn{2}{c}{ Female } & Male & & \\
\cline { 2 - 6 } & $n$ & $\%$ & $n$ & $\%$ & $n$ & $\%$ \\
\hline No stimulus & 31 & $63 \%$ & 18 & $37 \%$ & 49 & $34 \%$ \\
Neutral stimulus & 30 & $64 \%$ & 17 & $36 \%$ & 47 & $32 \%$ \\
Pleasant stimulus & 32 & $65 \%$ & 17 & $37 \%$ & 49 & $34 \%$ \\
Total & 93 & $64 \%$ & 52 & $36 \%$ & 145 & $100 \%$ \\
\hline
\end{tabular}

\section{Instruments}

Three tests comprised the battery for this study. The State-Trait Anxiety Inventory (STAI; Spielberger, Gorsuch, \& Lushene, 1970) was translated and adapted for Brazil by Biaggio, and Natalício (1979). The STAI has anxiety scales that are divided into trait (STAI-T) and state (STAI-S; Cronbach's $\alpha=.79$ ). The STAI enables distinctions between men and women (Giron, \& De Almeida, 2010).

The Focused Attention Test (Cambraia, 1967) evaluates the subject's ability to keep their attention focused on work performed during a given period of time (Cronbach's $\alpha=.73$ ).

The Personnel Selection Testing battery (PST-M battery; King, 2004) was translated and adapted by Piovani (2006). It consists of 11 subtests that are used to evaluate logical reasoning (judgment), memory, numeracy, perceptual skills (precision and perception), spatial ability (parts, blocks, and size), and vocabulary ability (fluency). It can be used in all areas in which a quick evaluation of various mental functions is required. For the purposes of this study, only the memory subtest was used to assess a visual exercise performed in daily life. The memory subtest determines the ability to retain and recall names, faces, and details in terms of association.

A pilot study was conducted with 34 students of both sexes to examine the emotional valence of the content of visual stimulation using the non-verbal pictorial Self-Assessment Manikin (SAM) scale. The content of the films was adequate for what was proposed, considering that the film with pleasant emotional content was considered as such by 12 of 16 participants, and the neutral film was identified as such by 17 of 18 participants.

\section{Procedures}

The application was taken in a public university. According to schedules provided by teachers, the tests were applied at the beginning of the classes or, sometimes, at the end of these. Following the heterogeneous elements of this study, the tests were diversified by selecting different courses and, in each one of them, applied in their own classrooms.

The students present in the class were invited to participate. After they agreed, they completed a demographic questionnaire and signed a consent form. Forty-nine participants completed three psychological tests that assessed attention, anxiety, and working memory without stimulus presentation. Forty-seven other students were exposed to a neutral visual stimulus followed by the application of the same three psychological tests. Forty-nine students were exposed to a pleasant visual stimulus and completed the same three psychological tests. The total duration of the application of the instruments in each class was $40 \mathrm{~min}$.

The neutral visual stimulus was a short $90 \mathrm{~s}$ video without audio that contained images of buildings and streets in a Brazilian city. This video had no images of people or animals. The pleasant visual stimulus was a scene from the movie Ice Age, which also lasted $90 \mathrm{~s}$ without audio. This scene contained one of the characters, the squirrel, that was engaged in an adventure behind a walnut, and funny things happen to him during this search.

\section{Statistical analysis}

The data were analyzed using SPSS for Windows, version 18.0. Analysis of variance was used to examine the variables of the three groups. Values of $p<0.05$ were considered statistically significant.

\section{Results}

With regard to memory measured by the PST battery, although the scores obtained by the subjects who were exposed to the pleasant visual stimulus were higher than the other groups, the difference was not statistically significant.

With regard to state anxiety measured by the STAI-S, the scores for the group of subjects who were exposed to the pleasant visual stimulus were not significantly different from the other groups. With regard to trait anxiety measured by the STAI-T, a significant decrease in scores was observed in the group that was exposed to the pleasant visual stimulus $(M=40.39)$.

With regard to attention measured by the Focused Attention Test, although the scores obtained by the subjects who were exposed to the pleasant visual stimulus 
were higher than the scores obtained by the other groups, the difference was not statistically significant. The means were 58.20 (pleasant stimulus), 55.49 (neutral stimulus), and 54.98 (no stimulus; Table 2).

With regard to gender differences, men had significantly lower results $(M=37.24, p=.01)$ in the test that assessed trait anxiety, indicating less anxiety symptoms, after being exposed to the pleasant stimulus compared with the women in the group $(M=42.06, p=.50$; Table 3$)$. The gender comparison indicated that women had significantly higher scores $(M=85.53)$ than men $(M=68.47)$ in the test that assessed working memory $(p=.02)$. No statistically significant differences were found in the comparison between genders for the Focused Attention Test.

Table 2. Anxiety, working memory, attention, and score for each group (mean $[M]$ and standard deviation $[S D]$ ).

\begin{tabular}{|c|c|c|c|c|c|c|c|}
\hline \multicolumn{8}{|c|}{ Group } \\
\hline Test & G1 & & G2 & & G3 & & $p$ \\
\hline & $\mathrm{M}$ & SD & $\mathrm{M}$ & SD & $\mathrm{M}$ & SD & \\
\hline Trait anxiety & 42.10 & 9.19 & 45.70 & 10.11 & 40.39 & 9.88 & 0.02 \\
\hline State anxiety & 42.53 & 8.54 & 45.40 & 9.63 & 43.27 & 8.40 & 0.26 \\
\hline $\begin{array}{l}\text { Working } \\
\text { memory }\end{array}$ & 75.80 & 14.23 & 73.62 & 22.41 & 78.31 & 18.16 & 0.46 \\
\hline Attention & 54.98 & 34.51 & 55.49 & 29.16 & 58.20 & 31.48 & 0.86 \\
\hline
\end{tabular}

Table 3. Description of STAI-T results obtained for men and women in Group 3 (pleasant stimulus).

\begin{tabular}{lllllll}
\hline \multirow{3}{*}{ STAI-T } & \multicolumn{7}{c}{ Sex } \\
\cline { 2 - 8 } & Male & & & Female & & \\
& $\mathrm{M}$ & $\mathrm{SD}$ & $\mathrm{p}$ & $\mathrm{M}$ & $\mathrm{SD}$ & $\mathrm{p}$ \\
& 37.24 & 9.43 & 0.01 & 42.6 & 9.85 & 0.5 \\
\hline
\end{tabular}

\section{Discussion}

The participants were not significantly different in the tests that assessed working memory, state anxiety, or attention. However, in the assessment of trait anxiety, a significant difference was found between the group that was exposed to the pleasant stimulus $(M=$ 40.39) and the other groups (no stimulus, $M=42.10$; neutral stimulus, $M=45.70 ; p=0.02$ ). These results corroborate previous findings that found that emotion is not the only factor that influences memory, and other factors may be important, such as prior knowledge and cognitive, social, and physiological factors, such as personal history, the environment, and culture (Christianson, 1992; Schacter, Kagan, \& Leichtman 1995). Davidson et al. (2000) and Davidson (2003; cited by Kosslyn et al., 2002) reported that an important focus of studies on emotional modulation is the analysis of individual variability in the processing of emotional stimuli. Affective bias is the most important feature of emotion because the same event can stimulate different emotional reactivity patterns in different individuals.

One important consideration in in the present study is that the pleasant stimulus was a small portion of a movie that was probably well known by most of the participants in the sample. This factor may have influenced the way the stimulus was perceived, whether eliciting excitation or not, because some of the participants may have empathy for the character, whereas others may not like it or did not form an opinion, thus generating different feelings or behaviors and directly influencing performance on the tests. Some authors have argued that emotional stimuli are given priority in the processing of attention and memory because of the level of excitation caused by the stimulus (Murphy et al., 2010).

Another factor that should be discussed is that a positive stimulus is more difficult to characterize than a negative stimulus. What may be enjoyable for one individual may not be enjoyable to another. Pinto (1998) suggested that negative emotional experiences are typically more distinct and intense than positive emotional experiences. According to Christianson and Safer (1996), this effect is attributable to the need to quickly identify and respond to negative and threatening stimuli. In a laboratory setting, inducing intense negative experiences is easier, faster, and more uniform than inducing positive experiences or negative emotional experiences of accidents and injuries or positive emotional experiences with anecdotes, descriptions, or erotic comedy movies. The classification of negative experiences also has greater uniformity than the classification of positive experiences (Pinto, 1998)One important point that should be noted is the fact that the instruments were applied at different school times (i.e., at the beginning, middle, and end), which may have influenced both the willingness to participate in the study and the mood of the participants in the sample.

The results showed that the sample of men had fewer symptoms of anxiety after being exposed to the pleasant stimulus, and women had superior results in the test that assessed working memory. We attempted to pair the number of men and women but could not because more women participated than men, a fact that usually occurs in other studies. Nonetheless, the difference in the number of men and women in the sample may have influenced the men's behavior.

With regard to state anxiety, we found that it was not influenced by the pleasant stimulus. Importantly, as noted by Vytal et al. (2012), anxiety can impair the ability to think and concentrate, suggesting that the interaction between emotion and cognition may elucidate the debilitating nature of pathological anxiety.

Although anxiety can be adaptive, it can also be debilitating by interfering with daily life and goaldirected behavior. Some of the cognitive problems that are prominent in anxiety are mediated by mechanisms of impaired attention. Anxious people complain of being easily distracted and have difficulty concentrating. Population-based studies have reported impairments in executive function and episodic memory in anxiety disorders (Airaksinen et al., 2005). One necessary consideration in the present study is that the participants were assessed at the end of the semester, a time when various scholastic tests and assessments are being conducted, thus possibly producing high levels of anxiety. The participants' baseline anxiety during the test period may have prevented the attainment of better results. 
Based on the results of the present study, we may conclude that the effect of pleasant emotional stimuli on cognition may be influenced by individual factors. The stimuli presented to the participants must be further reviewed and studied to verify their valence. Future studies should be conducted with pleasant visual stimuli to evaluate the influence of these stimuli on various aspects of cognition and emotion.

\section{References}

Airaksinen, E., Larsson, M., \& Forsell, Y. (2005). Neuropsychological functions in anxiety disorders in population-based samples: evidence of episodic memory dysfunction. Journal of Psychiatric Research, 39(2), 207-214.

Biaggio, A., Natalicio, L. F. \& Spielberger, C. D. - Desenvolvimento da Forma Experimental em Português do IDATE. Arquivos Brasileiros de Psicologia Aplicada 29: 33-44, 1977.

Buchanan, T. W., Denburg, N. L., Tranel, D., \& Adolphs, R. (2001). Verbal and nonverbal emotional memory following unilateral amygdala damage. Learning \& Memory, 8, 326-335.

Cahill, M. (2000). Tonal diversity in languages of Papua New Guinea. Paper presented at the Tone and Pitch Accent Conference, Tromsø, Norway, June 5-7, 2000.

Cambraia, S. V. (1967). Teste atenção concentrada. São Paulo: Vetor.

Christianson, S. Å., \& Safer, M. A. (1996). Emotional events and emotions in autobiographical memories. In D. C. Rubin (Ed.), Remembering our past: studies in autobiographical memory (pp. 218-243). Cambridge: Cambridge University Press.

Christianson, S. A. (1992). The handbook of emotion and memory: research and theory. Hillsdale, $\mathrm{NJ}$ : Erlbaum.

Compton, R. J. (2003). The interface between emotion and attention: a review of evidence from psychology and neuroscience. Behavioral and Cognitive Neuroscience Reviews, 2, 115-129.

Damásio, A. R. (2000). A second chance for emotion. In R.D. Lane, \& L. Nadel (Eds.), Cognitive neuroscience of emotion (pp. 12-23). New York: Oxford University Press.

Davidson, R. J. (2003). Affective neuroscience and psychophysiology: toward a synthesis. Psychophysiology, 40(5), 655-665.

Davidson, R. J., Jackson D. C., \& Kalin, N. H. (2000). Emotion, plasticity, context and regulation: perspectives from affective neuroscience. Psychological Bulletin, 126, 890-909.

Davis , M., \& Whalen , P. J. (2001). The amygdala: vigilance and emotion. Molecular Psychiatry, 6, 13-34.

Dolan, R. J. (2002). Emotion, cognition, and behavior. Science, 298(5596), 1191-1194.

Dreisbach, G., \& Goschke, T. (2004). How positive affect modulates cognitive control: reduced perseveration at the cost of increased distractibility. Journal of Experimental Psychology, 30(2), 343-353.

Giron, P. R, \& De Almeida, R. M. M. (2010). Influence of aversive visual stimulation on attention, working memory, and anxiety in university students. Psychology and Neuroscience, 3 (1), 109115.

Heilman, K. M. (1997). The neurobiology of emotional experience. Journal of Neuropsychiatry and Clinical Neurosciences, 9, 439-448.

Kensinger, E. A., \& Corkin, S. (2003). Memory enhancement for emotional words: are emotional words more vividly remembered than neutral words? Memory \& Cognition, 31(8), 1169-1180.
King, J. E. (2004) The Personnel Selection Testing battery (PST-M battery). USA

Kosslyn, S. M., Cacioppo, J.T ., Davidson, R. J., Hugdahl, K., Lovallo, W. R., Spiegel, D., \& Rose, R. (2002). Bridging psychology and biology: the analysis of individuals in groups. American Psychologist, 57, 341-351.

LaBar, K. S., \& Phelps, E. A. (1998). Arousal-mediated memory consolidation: role of the medial temporal lobe in humans. Psychological Science, 9(6), 490-493.

Lang, P. J. (1995). The emotion probe: studies of motivation and attention. American Psychologist, 50(5), 372-385.

Lowenstein, G., \& Lerner, J. S. (2003). The role of affect in decision making. In R. Davidson, K. Scherer, \& H. Goldsmith (Eds.). Handbook of affective science, pp. 619-642. New York: Oxford University Press.

Lazarus, R. S. (1993). "Why We Should Think of Stress as a Sugset of Emotion", in L. Goldberg and S. Bredmitz (Eds), Handbook of Stress, Theoritical and Clinical Aspects (pp. 21-39). New York: Free Press.

Moll, J., Souza, R., Miranda, J., Bramati I., Veras, R., \& Magalhães, A. C. (2001). Efeitos distintos da valência emocional positiva e negativa na ativação cerebral. Revista Brasileira de Psiquiatria, 23(I), 42-45.

Murphy, F. C., Hill, E. L., Ramponi, C., Calder, A. J., \& Barnard P. J. (2010). Paying attention to emotional images with impact. Emotion, 10(5), 605-614.

Oaksford, M., Morris, F., Grainger, B., \& Williams, J. M. (1996). Mood, reasoning, and central executive processes. Journal of Experimental Psychology: Learning, Memory, and Cognition, 22, 477-492.

Ochsner, K. N. (2000). Are affective events richly recollected or simply familiar? The experience and process of recognizing feelings past. Journal of Experimental Psychology: General, 129(2), 242-261.

Pergher, G. K., Grassi-Oliveira, R., Ávila, L. M., \& Stein, L. M. (2006). Memória, humor e emoção. Revista de Psiquiatria do Rio Grande do Sul, 28(1), 61-68.

Piovani, C. (2006) Bateria TSP. São Paulo: Edites, s/d.

Phillips, L. H., Bull, R., Adams, E., \& Fraser, L. (2002). Positive mood and executive function: evidence from Stroop and Fluency Tasks. Emotion, 2(1), 12-22.

Pinto, A.C. (1998). O impacto das emoç̃es na memória: alguns temas em análise. Psicologia, Educação e Cultura, 2(2), 215-240.

Rolls, E. T. (1999). The brain and emotion. New York: Oxford University Press.

Schacter, D. L., Kagan, J., \& Leichtman, M. D. (1995). True and false memories in children and adults: A cognitive neuroscience perspective. Psychology, Public Policy, and Law, 1, 411-428.

Shohamy, D., \& Adcock, R. A. (2010). Dopamine and adaptive memory. Trends in Cognitive Sciences, 14(10), 464-472.

Souza, G. I. L., Pereira, M. G., Oliveira, L., Pinheiro W. M., \& Volchan, E. (2005). Influência do humor e do traço afetivo sobre o tempo de reação para detecção de estímulo visual. Arquivos Brasileiros de Psiquiatria, Neurologia e Medicina Legal, 99(2), 8-14.

Spielberger, C. D., Gorsuch, R. L., \& Lushene, R. E. (1970). Manual for the State-Trait Anxiety Inventory. Palo Alto, CA: Consulting Psychologists Press.

Vytal, K., Cornwell, B., Arkin, N., \& Grillon, C. (2012). Describing the interplay between anxiety and cognition: from impaired performance under low cognitive load to reduced anxiety under high load. Psychophysiology, 49(6), 842-852. 